\title{
RFID Smart Shelf With Confined Detection Volume at UHF
}

\author{
Carla R. Medeiros, Jorge R. Costa, Member, IEEE, and Carlos A. Fernandes, Senior Member, IEEE
}

\begin{abstract}
This letter presents a smart shelf configuration for radio frequency identification (RFID) application. The proposed shelf has an embedded leaking microstrip transmission line with extended ground plane. This structure, when connected to an RFID reader, allows detecting tagged objects in close proximity with proper field confinement to avoid undesired reading of neighboring shelves. The working frequency band covers simultaneously the three world assigned RFID subbands at UHF[AU: Please define UHF]. The concept is explored by full-wave simulations and it is validated with thorough experimental tests.
\end{abstract}

Index Terms-Microstrip antennas, near fields, radio frequency identification (RFID), reader antenna, smart shelf.

\section{INTRODUCTION}

$\mathbf{R}$ ADIO frequency identification (RFID) technology enables detection and recognition of tagged items, which associate an object to an univocal identification code. In a preliminary development stage, RFID technology was mainly applied to access control and inventory management. However, the advantages of this technology rapidly extended its applicability to other areas like object location.

RFID technology world assigned frequency bands range from HF up to microwaves. At UHF, the usable band ranges from 860 to $960 \mathrm{MHz}$. In fact, in order to be compatible with mobile communications assigned bands, the International Telecommunications Union (ITU) has allocated for RFID three different intervals of the UHF band for different world regions. In general, UHF RFID tags cover the widest range of applications and its unit cost is the lowest. Tag cost is a critical issue for retail store RFID deployment; therefore, this work focuses on the UHF RFID band.

The so-called RFID smart shelf is a regular shelf with an associated antenna reader, eventually embedded in the shelf, which

Manuscript received August 03, 2008; revised September 14, 2008. First published October 31, 2008; current version published January 16, 2009. Patent pending PT 104121.

C. R. Medeiros is with the Instituto de Telecomunicações, Av. Rovisco Pais, 1049-001 Lisboa, Portugal (e-mail: Carla.Medeiros@1x.it.pt).

J. R. Costa is with the Instituto de Telecomunicações, 1049-001 Lisboa, Portugal, and also with the Instituto Superior de Ciências do Trabalho e da Empresa, 1649-026 Lisboa, Portugal (e-mail: Jorge.Costa@1x.it.pt).

C. A. Fernandes is with the Instituto de Telecomunicações, and with Instituto Superior Técnico, 1049-001 Lisboa, Portugal (e-mail: Carlos.Fernandes@1x.it. pt).

Color versions of one or more of the figures in this letter are available online at http://ieeexplore.ieee.org

Digital Object Identifier 10.1109/LAWP.2008.2008672 ideally allows to univocally detecting all the tagged items located on that shelf only. Extending this concept to every shelf in a store makes it possible to automatically locate and inventory every item. The main challenges are as follows:

1) to ensure that the shelf antenna reading capability is confined to the volume above that shelf and does not extend to contiguous shelves;

2) the solution must be compatible with the existing passive UHF tags, which are much cheaper than active tags, but have shorter range; it is desirable that the shelf antenna operation band is wide enough to cover the three RFID world UHF bands;

3) the shelf antenna must be simple enough for low-cost mass production and easily adaptable to different shelf sizes and types.

Although some solutions can be found in the literature for RFID smart tables and shelves, these operate at the HF band [1], [2] and no smart-shelf solution is reported for UHF. The main difference is that at HF the energy coupling between the reader antenna and the tag is essentially made through the magnetic field. A very common reader antenna configuration is a loop antenna [1], [2]. Good coupling requires close proximity between reader antenna and the tag, so detection confinement is inherently ensured. At UHF, readers are equipped with antennas such as patch antennas [3] and energy coupling to the tag antenna is made through electromagnetic propagating waves. Considering wavelengths in the order of $30 \mathrm{~cm}$, it is difficult to limit the antenna radiation exactly to the shelf boundary without resorting to costly metal or absorbing shields. For instance, in a different application reported in [4], a tunnel system is used to confine the radiation from three near field patches used to identify boxes.

In this letter, a very simple, effective, and low-cost solution is proposed for the RFID smart shelf concept at UHF complying with all the above itemized specifications. The shelf incorporates a leaking microstrip line with an extended ground plane. Microstrips are most commonly used in microwave circuits as transmission lines, primarily due to its simple fabrication and integration with other devices [5]. Conversely to its main applicability, the designed shelf exploits microstrip line leaking fields (undesirable in microwave circuits) to enable RFID in the near range. The proposed solution can be easily manufactured and further embedded or attached to shelf structures. When combined with commercial RFID readers (Fig. 1), it enables proximity tag detection through electric field activation. Although the proposed concept can be generalized to other near-field RFID applications at UHF, this letter focuses on smart shelves for bookstores and libraries. A single reader can be used with multiple smart shelves using a standard electronically actuated RF switch to sequentially read each shelf in the 


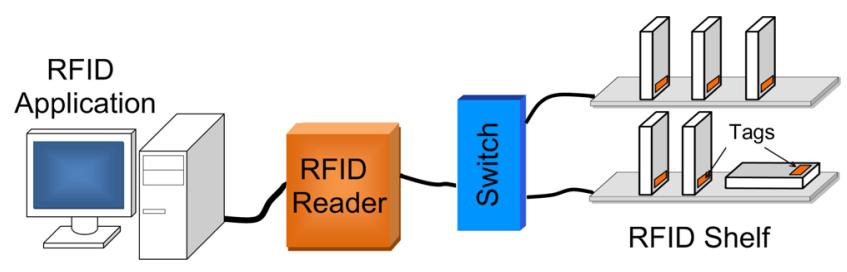

Fig. 1. RFID system.

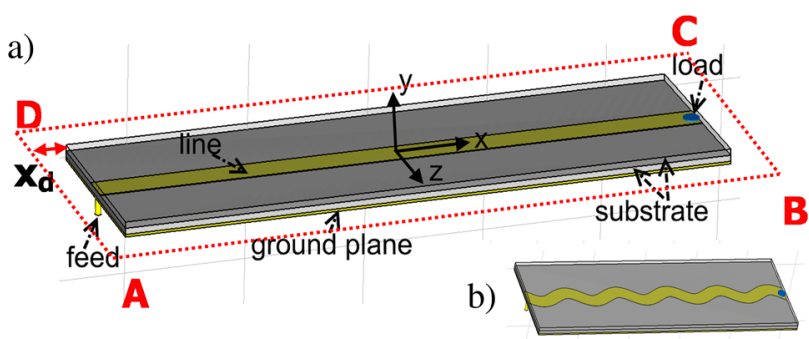

Fig. 2. Smart shelf configuration: (a) straight line; (b) meandered line.

store. In this way, the required RF power output from the reader (about $1 \mathrm{~W}$ ) is enough to read an arbitrary number of shelves in the store. A shelf prototype was fabricated and reading score was evaluated at UHF both for isolated tags and for tags within books. The results of the experimental tests agree very well with simulations and show the effectiveness of the solution.

\section{SHELF DESCRIPTION}

\section{A. Shelf Basic Configuration}

The embedded microstrip line extends across the shelf length; it is probe fed in one end and it is terminated with a matched load (a resistor). In microstrip lines, the main electric field lines flow from the metal strip to the ground plane, through the substrate layer, while some radiation leaks to the air along the lateral edge of the strip. To enhance this radiation and ensure a reasonable tag detection range, the line is printed on a very low permittivity dielectric; additionally, it may be slightly meandered. The transmission line can be easily embedded in dielectric shelves or attached to layers of any material. In the case of metallic shelves, the shelf itself may act as the line's ground plane.

An example of shelf structure is shown in Fig. 2. Its overall dimensions are $30 \mathrm{~cm} \times 100 \mathrm{~cm}$, typical for bookshelves. A 1 ErmEthick styrofoam $\left(\varepsilon_{r} \approx 1.05\right)$ substrate layer is used. The transmission line is centered on the shelf and its width is $5 \mathrm{~cm}$ so that a $50 \boxminus \Omega$ impedance line is obtained. The ground plane is extended up to the shelf edges. The structure is fed by $50 F \Omega$ coaxial cable at one end of the line and terminated by $50 E \Omega$ load. An additional layer of styrofoam substrate is added on top of the transmission line to increase mechanical stability and avoid direct contact between the books and the microstrip. Full wave simulations were done using CST Microwave Studio [6]. The model is shown in Fig. 2(a), where the transmission line is oriented along the $x$-axis direction.

\section{B. Shelf Near-Field Distribution}

Near-field calculations were performed for the UHF band $(866 \mathrm{MHz})$ to evaluate the shelf performance. The line is fed
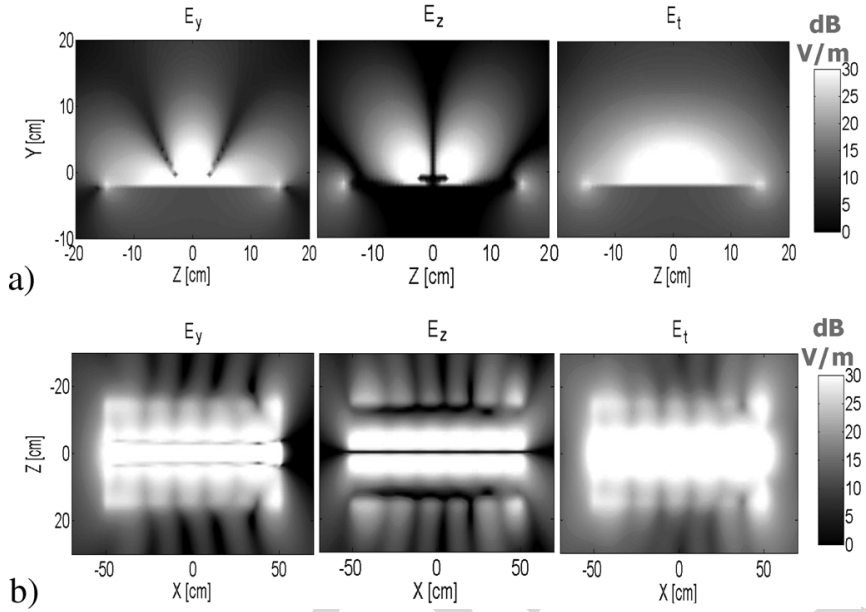

Fig. 3. Near-field components: (a) $X=0$ plane; (b) $Y=0$ plane (shelf surface).

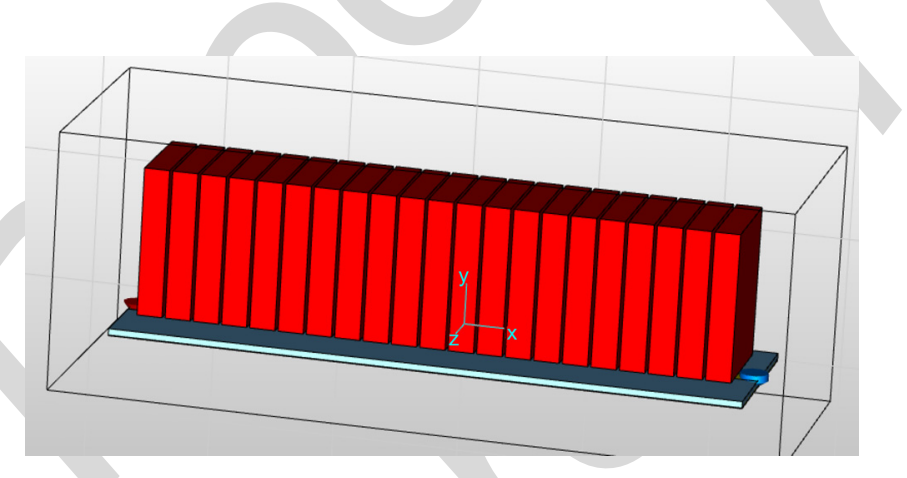

Fig. 4. Simulation model of the shelf with 21 books on top.

by $1 \mathrm{~W}$, the same power that is available from the reader used in Section III. The near-field distribution at the $Y Z$ and $X Z$ planes is shown in Fig. 3. The results at the $X=0$ plane shown in Fig. 3 confirm, as could be expected, that the dominant electric field components are $E_{y}$ and $E_{z}$ although the latter is very low end exactly $z=0$, the center of the shelf (conversely to the vertical component $E_{y}$ ). The $E_{x}$ component is very low and it is not shown in Fig. 3. It was experimentally verified that the tag detection level corresponds in this figure approximately to $20 \mathrm{dBV} / \mathrm{m}$. It is noted that the fields are reasonably confined to the shelf near zone.

The influence of the books on the shelf was also modeled by CST simulation (Fig. 4). The 21 books were modeled as $0.5 \mathrm{gcm} \boxminus$ spaced blocks of $\varepsilon_{r}=2.3$ dielectric, with $4 \mathrm{~g} \mathrm{~cm}$ width, $23 \mathrm{Ecm}$ height, and $18 \mathrm{Em}$ depth. The calculated field distribution is shown in Fig. 5. The field intensity is enhanced everywhere in the shelf. For linear polarized tags, detection is possible when they are oriented along $y$ - and $z$-axis directions. The field confinement is very neat especially for the $E_{z}$ component.

Simulated results demonstrate that a single transmission line is sufficient for detection of tagged books on a $30 \mathrm{gcm} \boxminus$ width shelf. However, for wider shelves, multiple lines or a lightly meandered line path [see Fig. 2(b)] may be required to ensure appropriate illumination of the whole shelf. In the latter case, the $E_{x}$ component is further enhanced, reducing tag polarization discrimination. Also, the maximum detection height is increased without affecting the overall tag detection score. This 
a)
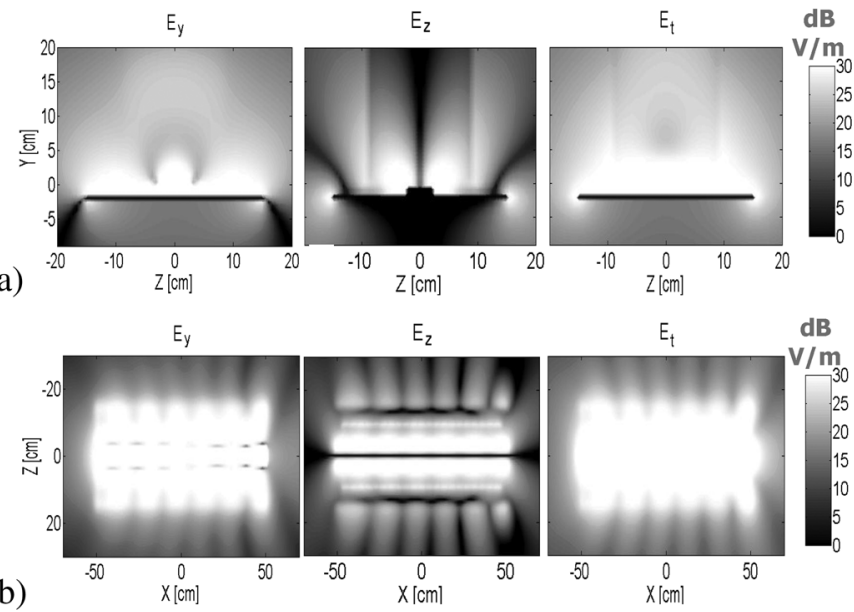

Fig. 5. Near-field components of the shelf with 21 books: (a) $X=0$ plane; (b) $Y=0$ plane (shelf surface)

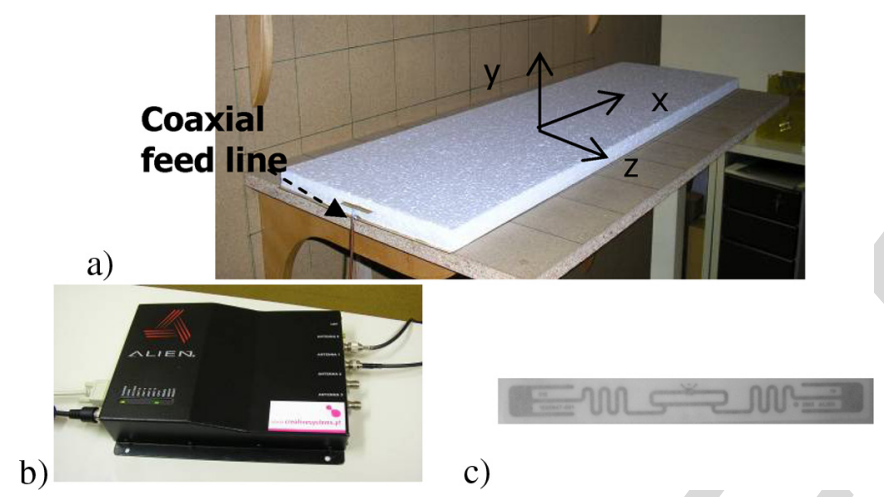

Fig. 6. Test environment: (a) fabricated shelf prototype; (b) ALIEN ALR-8800 reader; (c) ALIEN ALN-9540- "Squiggle" tag.

height is determined by the shape and lateral extent of the strip skewed path.

\section{EXPERIMENTAL RESULTS}

Fig. 6 shows the test environment used to evaluate the effectiveness of the proposed structure. The smart shelf is mounted on top of a preexisting wood structure [see Fig. 6(a)]. The ALIEN-ALR-8800 reader [7] in Fig. 6(b) is used to feed the proposed structure and its native software is used for score reading. The tags are ALIEN-ALN-9540_-"Squiggle" GEN 2 tag [7] [Fig. 6(c)]. The selected tag, with $9.7 \mathrm{~cm} \times 1.1 \mathrm{~cm}$ total size, is linear polarized and operates from 860 up to 920 MHz.

Measured and simulated input return loss of the embedded straight transmission line is presented in Fig. 7, both for the empty shelf and when it is fully loaded with 21 books. The transmission line is terminated with a matched load. The agreement between simulation and measurements is satisfactory. It is noted that when the 21 books are present, the line input return loss improves slightly, suggesting that more power is drained out from the line in this case.

Electric field components were measured near the empty shelf with the embedded microstrip line. The Agilent E8361A network analyzer was used in this test with a near-field probe a)

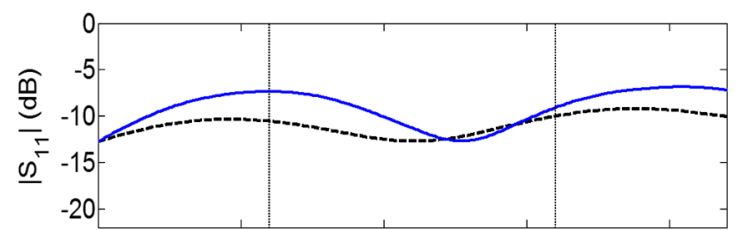

b)

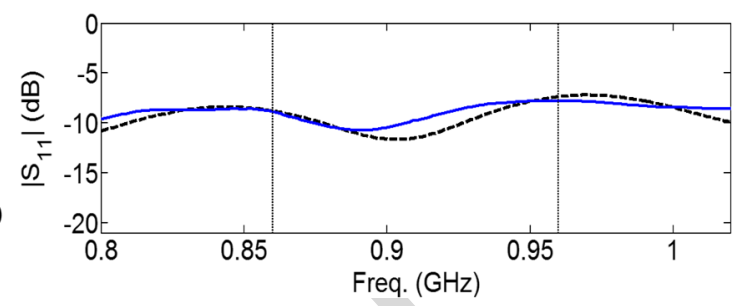

Fig. 7. Measured ( - ) and simulated (---) input impedance: (a) empty shelf; (b) shelf with 21 books.

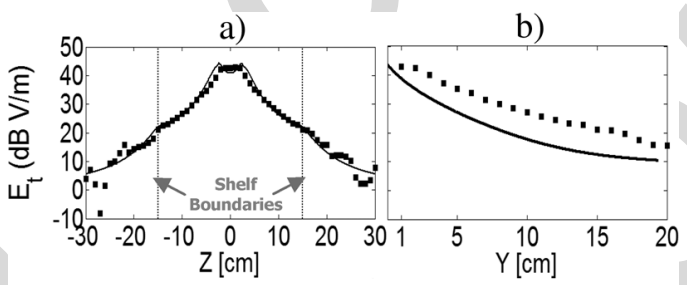

c)

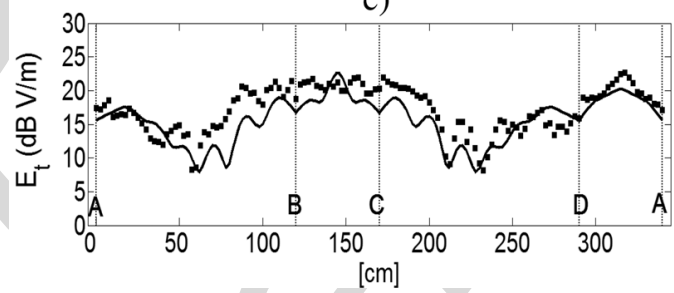

Fig. 8. Simulated $(-)$ and measured $(\cdots)$ total electrical near field in a shelf without books: (a) $x=0 \mathrm{~cm}, z=3 \mathrm{~cm}$ cut; (b) $y=0 \mathrm{~cm}, x=0 \mathrm{~cm}$ cut; (c) $y=0 \mathrm{~cm}, 10 \mathrm{~cm}$ away from the shelf over the ABCDA contour shown in Fig. 2(a).

scanning across two cut planes and along the shelf outside perimeter ABCDA (Fig. 2). Fig. 8 shows measured results superimposed on simulations: the agreement is quite good. Fig. 8(a) corresponds to $z$-axis scan $(x=0, y=0)$ and shows fast field decay outside the shelf, below the $20 \mathrm{AdBV} / \mathrm{m}$ tag detection level. Fig. 8(b) corresponds to vertical coordinate scan at $(x=0, z=3)$ and anticipates tag detection up to 7 $\mathrm{cm}$. Fig. 8(c) corresponds to the closed contour of Fig. 2(a) for $x_{d}=10 \mathrm{~cm}$ : total electric field is maximum near the line feed and termination points, however field level is mainly below 20 $\mathrm{dBV} / \mathrm{m}$. In all cases, the experimental results follow quite well the simulation predictions.

For experimental confirmation of the RFID tag detection performance, the volume above the shelf was divided into a virtual lattice and an isolated tag was positioned at each node, according to prescribed orientations; the reading score was registered at each tag position.

The reading score was $100 \%$ for $z$-axis tag orientation over the shelf surface (Fig. 9). Although the $E_{z}$ component intensity is very low at the center of the microstrip line, this effect is very localized and does not deter reliable tag detection. The height above the shelf surface where the tag can be detected is 


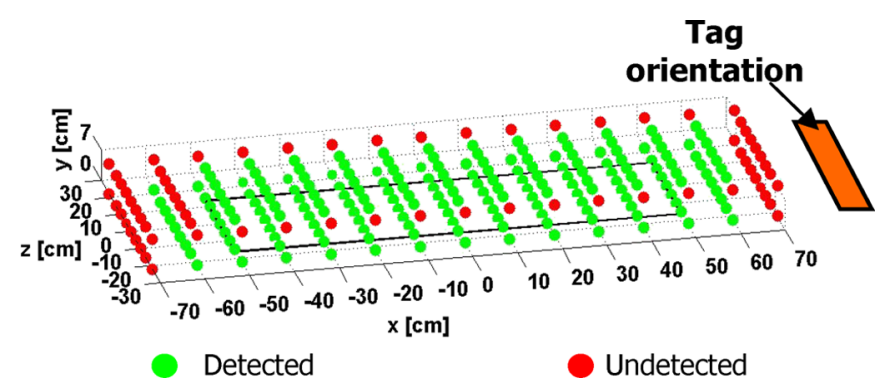

Fig. 9. Results of experimental detection of standalone tag positioned at each represented point, with $z$-axis orientation, without books.

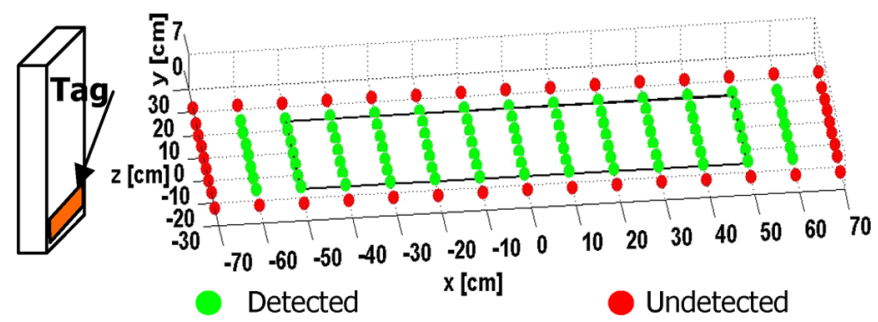

Fig. 10. Experimental detection of tags within books, oriented along $z$-axis.

controlled by adjusting the RF signal attenuation on the reader. For the straight microstrip line and no attenuation at the reader, $100 \%$ detection score is obtained up to $7 \mathrm{~cm}$ above the shelf surface, without spurious detection of tags placed on the shelf above or on the shelf below. The t $\mathrm{rm}$ maximum detection height is sufficient for the bookshelf application. Experimental results show that tag detection is obtained also for $x$-axis orientation in the regions above the microstrip line, where the other electric field components are also significant.

Similar tests were performed with the tags inserted into books, oriented along the $z$-axis. This is the preferred orientation to take advantage of the predominant and well confined $E_{z}$ field component. Simulations and experiment show that the presence of the book enhances the field intensity, favoring tag detection. Measurements were performed with a single book, with clusters of books, and with fully loaded shelf. Results for a single book are shown in Fig. 10. Each dot corresponds to each test position of the center of the book. It is seen that book detection is reasonably contained within the limits of the shelf. Experiments confirm that the lowest the tag placed inside the book is, the more reliable is the shelf reading capability. Similar results are obtained for fully loaded shelf.

Although the shelf design is intended for $z$-axis tag orientation, experiments showed that near $100 \%$ detection score is still possible when the tag inside the book is rotated in the $y-z$ plane. Even for the extreme $y$-axis tag orientation inside the book, detection is possible over most of the shelf surface, except for a region about $7 \mathrm{~cm}$ away from the center of the shelf [observable in Fig. 3(a)] where the intensity $E_{y}$ component is low and reading score may degrade.

So, books should be preferably placed vertically in the shelves with their tags at the bottom, oriented along the $z$-axis (transversal to the shelf). However, some flexibility is allowed in the tag orientation and height and detection is obtained as well for other book orientations on the shelf. These features favor practical use of the proposed solution.

\section{CONCLUSION}

This letter presents a new RFID low-cost smart shelf for book identification at UHF with proper confinement of the electromagnetic fields to avoid detection of books located outside the interrogated shelf, without the need of EM isolation barriers. The proposed approach uses the leaking fields of shaped microstrip transmission lines to enable proximity tags detection.

A prototype of the shelf was fabricated and tested using commercial reader and tags. Results demonstrate a very good match between experimental reading score and the simulated nearfield distribution; $100 \%$ reading score was achieved with the books and tags in the preferred orientations, but other orientations are also allowed which makes the proposed solution adequate for practical application on retail stores.

The presented smart shelf structure can be redesigned, without frequency restriction, to adjust for different shelf sizes and requirements. The transmission line circuit can be embedded on the shelf or mounted on top, for example, when metallic shelves are present, or above a preexisting structure.

Even though the presented work is focused on shelves and books, the concept has been successfully tested for other applications where tight detection confinement at UHF is required. For example, using meandered lines, it is possible to extend the detection height level and ensure detection for all tag orientations, which is attractive for shop cashier conveyors for automatic RFID reading of the product information as it slides over the device. It can be used also at isolated RFID reader points in the store. In summary, it can replace some functions that are presently performed with optical bar code manual readers, enabling a complete RFID-based solution in retail shop environment.

\section{ACKNOWLEDGMENT}

The authors would like to thank Creativesystems for discussions about the motivation and system specifications and for providing the RFID tags and reader equipment. They would also like to thank V. Fred and C. Brito for prototype fabrication and A. Almeida for prototype measurements.

\section{REFERENCES}

[1] X. Qing and Z. N. Chen, "Proximity effects of metallic environments on high frequency RFID reader antenna: Study and applications," IEEE Trans. Antennas Propag., vol. 55, no. 11, pp. 3105-3111, Nov. 2007.

[2] A. Cai, X. M. Qing, and Z. N. Chen, "High frequency RFID smart table antenna," Microw. Opt. Technol. Lett., vol. 49, no. 9, pp. 2074-2076, Sep. 2007.

[3] J. M. Lee, N. S. Kim, and C. S. Pyo, "A circular polarized metallic patch antenna for RFID reader," in Proc. Asia-Pacific Conf. Commun., Australia, Oct. 2005, pp. 116-118.

[4] Z.-M. Liu and R. R. Hillegass, "A 3 patch near field antenna for conveyor bottom read in RFID sortation application," in Proc. IEEE Antenna Propag. Soc. Int. Symp., Jul. 2006, pp. 1043-1046.

[5] D. M. Pozar, Microwave Engineering, 2nd ed. New York: Wiley, 1998.

[6] CST- Computer Simulation Tech. May 2008 [Online]. Available: http:// www.cst.com/

[7] ALIEN Technology May 2008 [Online]. Available: http://www.alientechnology.com 


\title{
RFID Smart Shelf With Confined Detection Volume at UHF
}

\author{
Carla R. Medeiros, Jorge R. Costa, Member, IEEE, and Carlos A. Fernandes, Senior Member, IEEE
}

\begin{abstract}
This letter presents a smart shelf configuration for radio frequency identification (RFID) application. The proposed shelf has an embedded leaking microstrip transmission line with extended ground plane. This structure, when connected to an RFID reader, allows detecting tagged objects in close proximity with proper field confinement to avoid undesired reading of neighboring shelves. The working frequency band covers simultaneously the three world assigned RFID subbands at UHF[AU: Please define $U \boldsymbol{H F}$ ]. The concept is explored by full-wave simulations and it is validated with thorough experimental tests.
\end{abstract}

Index Terms-Microstrip antennas, near fields, radio frequency identification (RFID), reader antenna, smart shelf.

\section{INTRODUCTION}

$\mathbf{R}$ ADIO frequency identification (RFID) technology enables detection and recognition of tagged items, which associate an object to an univocal identification code. In a preliminary development stage, RFID technology was mainly applied to access control and inventory management. However, the advantages of this technology rapidly extended its applicability to other areas like object location.

RFID technology world assigned frequency bands range from HF up to microwaves. At UHF, the usable band ranges from 860 to $960 \mathrm{MHz}$. In fact, in order to be compatible with mobile communications assigned bands, the International Telecommunications Union (ITU) has allocated for RFID three different intervals of the UHF band for different world regions. In general, UHF RFID tags cover the widest range of applications and its unit cost is the lowest. Tag cost is a critical issue for retail store RFID deployment; therefore, this work focuses on the UHF RFID band.

The so-called RFID smart shelf is a regular shelf with an associated antenna reader, eventually embedded in the shelf, which

Manuscript received August 03, 2008; revised September 14, 2008. First published October 31, 2008; current version published January 16, 2009. Patent pending PT 104121.

C. R. Medeiros is with the Instituto de Telecomunicações, Av. Rovisco Pais, 1049-001 Lisboa, Portugal (e-mail: Carla.Medeiros@lx.it.pt).

J. R. Costa is with the Instituto de Telecomunicações, 1049-001 Lisboa, Portugal, and also with the Instituto Superior de Ciências do Trabalho e da Empresa, 1649-026 Lisboa, Portugal (e-mail: Jorge.Costa@1x.it.pt).

C. A. Fernandes is with the Instituto de Telecomunicações, and with Instituto Superior Técnico, 1049-001 Lisboa, Portugal (e-mail: Carlos.Fernandes@1x.it. pt).

Color versions of one or more of the figures in this letter are available online at http://ieeexplore.ieee.org

Digital Object Identifier 10.1109/LAWP.2008.2008672 ideally allows to univocally detecting all the tagged items located on that shelf only. Extending this concept to every shelf in a store makes it possible to automatically locate and inventory every item. The main challenges are as follows:

1) to ensure that the shelf antenna reading capability is confined to the volume above that shelf and does not extend to contiguous shelves;

2) the solution must be compatible with the existing passive UHF tags, which are much cheaper than active tags, but have shorter range; it is desirable that the shelf antenna operation band is wide enough to cover the three RFID world UHF bands;

3) the shelf antenna must be simple enough for low-cost mass production and easily adaptable to different shelf sizes and types.

Although some solutions can be found in the literature for RFID smart tables and shelves, these operate at the HF band [1], [2] and no smart-shelf solution is reported for UHF. The main difference is that at HF the energy coupling between the reader antenna and the tag is essentially made through the magnetic field. A very common reader antenna configuration is a loop antenna [1], [2]. Good coupling requires close proximity between reader antenna and the tag, so detection confinement is inherently ensured. At UHF, readers are equipped with antennas such as patch antennas [3] and energy coupling to the tag antenna is made through electromagnetic propagating waves. Considering wavelengths in the order of $30 \mathrm{~cm}$, it is difficult to limit the antenna radiation exactly to the shelf boundary without resorting to costly metal or absorbing shields. For instance, in a different application reported in [4], a tunnel system is used to confine the radiation from three near field patches used to identify boxes.

In this letter, a very simple, effective, and low-cost solution is proposed for the RFID smart shelf concept at UHF complying with all the above itemized specifications. The shelf incorporates a leaking microstrip line with an extended ground plane. Microstrips are most commonly used in microwave circuits as transmission lines, primarily due to its simple fabrication and integration with other devices [5]. Conversely to its main applicability, the designed shelf exploits microstrip line leaking fields (undesirable in microwave circuits) to enable RFID in the near range. The proposed solution can be easily manufactured and further embedded or attached to shelf structures. When combined with commercial RFID readers (Fig. 1), it enables proximity tag detection through electric field activation. Although the proposed concept can be generalized to other near-field RFID applications at UHF, this letter focuses on smart shelves for bookstores and libraries. A single reader can be used with multiple smart shelves using a standard electronically actuated RF switch to sequentially read each shelf in the 


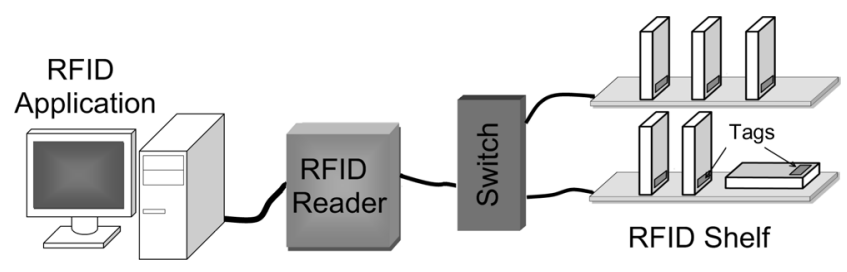

Fig. 1. RFID system.

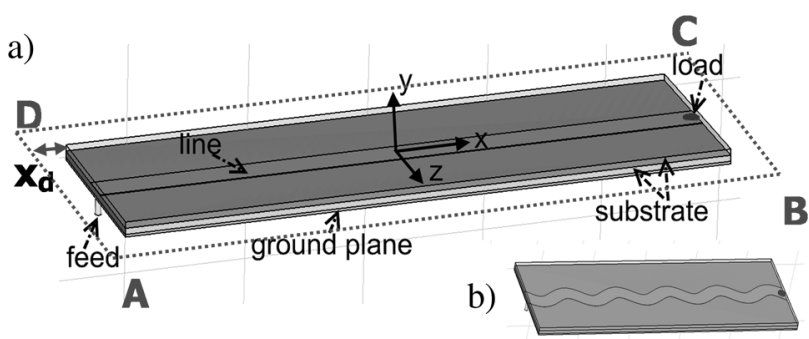

Fig. 2. Smart shelf configuration: (a) straight line; (b) meandered line.

store. In this way, the required RF power output from the reader (about $1 \mathrm{~W}$ ) is enough to read an arbitrary number of shelves in the store. A shelf prototype was fabricated and reading score was evaluated at UHF both for isolated tags and for tags within books. The results of the experimental tests agree very well with simulations and show the effectiveness of the solution.

\section{SHELF DESCRIPTION}

\section{A. Shelf Basic Configuration}

The embedded microstrip line extends across the shelf length; it is probe fed in one end and it is terminated with a matched load (a resistor). In microstrip lines, the main electric field lines flow from the metal strip to the ground plane, through the substrate layer, while some radiation leaks to the air along the lateral edge of the strip. To enhance this radiation and ensure a reasonable tag detection range, the line is printed on a very low permittivity dielectric; additionally, it may be slightly meandered. The transmission line can be easily embedded in dielectric shelves or attached to layers of any material. In the case of metallic shelves, the shelf itself may act as the line's ground plane.

An example of shelf structure is shown in Fig. 2. Its overall dimensions are $30 \mathrm{~cm} \times 100 \mathrm{~cm}$, typical for bookshelves. A 1 -cm-thick styrofoam $\left(\varepsilon_{r} \approx 1.05\right)$ substrate layer is used. The transmission line is centered on the shelf and its width is $5 \mathrm{~cm}$ so that a $50-\Omega$ impedance line is obtained. The ground plane is extended up to the shelf edges. The structure is fed by $50-\Omega$ coaxial cable at one end of the line and terminated by $50-\Omega$ load. An additional layer of styrofoam substrate is added on top of the transmission line to increase mechanical stability and avoid direct contact between the books and the microstrip. Full wave simulations were done using CST Microwave Studio [6]. The model is shown in Fig. 2(a), where the transmission line is oriented along the $x$-axis direction.

\section{B. Shelf Near-Field Distribution}

Near-field calculations were performed for the UHF band $(866 \mathrm{MHz})$ to evaluate the shelf performance. The line is fed
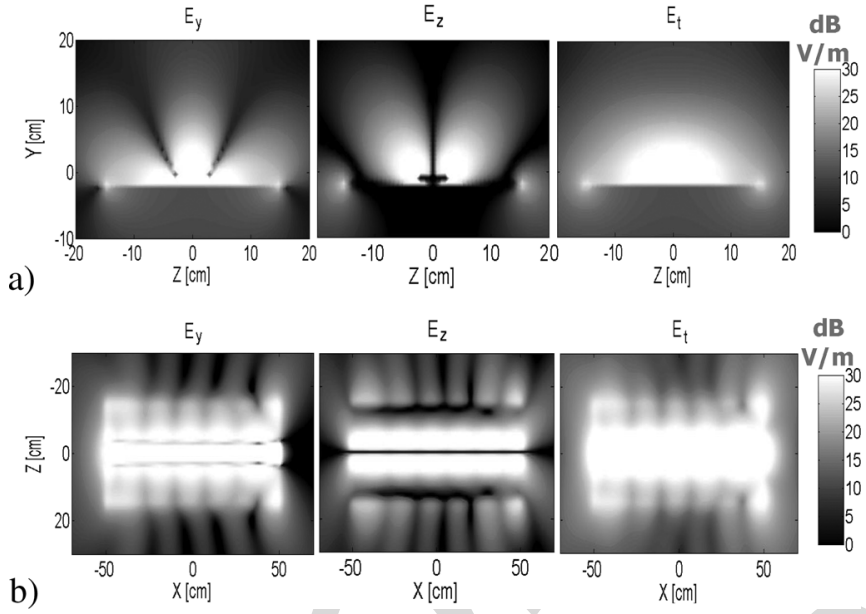

Fig. 3. Near-field components: (a) $X=0$ plane; (b) $Y=0$ plane (shelf surface).

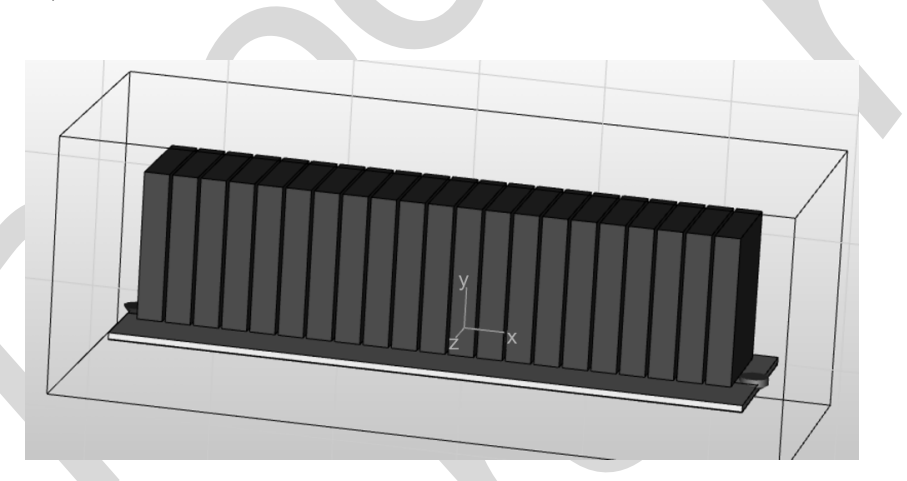

Fig. 4. Simulation model of the shelf with 21 books on top.

by $1 \mathrm{~W}$, the same power that is available from the reader used in Section III. The near-field distribution at the $Y Z$ and $X Z$ planes is shown in Fig. 3. The results at the $X=0$ plane shown in Fig. 3 confirm, as could be expected, that the dominant electric field components are $E_{y}$ and $E_{z}$ although the latter is very low and exactly $z=0$, the center of the shelf (conversely to the vertical component $E_{y}$ ). The $E_{x}$ component is very low and it is not shown in Fig. 3. It was experimentally verified that the tag detection level corresponds in this figure approximately to $20 \mathrm{dBV} / \mathrm{m}$. It is noted that the fields are reasonably confined to the shelf near zone.

The influence of the books on the shelf was also modeled by CST simulation (Fig. 4). The 21 books were modeled as 0.5 -cm-spaced blocks of $\varepsilon_{r}=2.3$ dielectric, with 4-cm width, $23-\mathrm{cm}$ height, and $18-\mathrm{cm}$ depth. The calculated field distribution is shown in Fig. 5. The field intensity is enhanced everywhere in the shelf. For linear polarized tags, detection is possible when they are oriented along $y$ - and $z$-axis directions. The field confinement is very neat especially for the $E_{z}$ component.

Simulated results demonstrate that a single transmission line is sufficient for detection of tagged books on a 30-cm-width shelf. However, for wider shelves, multiple lines or a lightly meandered line path [see Fig. 2(b)] may be required to ensure appropriate illumination of the whole shelf. In the latter case, the $E_{x}$ component is further enhanced, reducing tag polarization discrimination. Also, the maximum detection height is increased without affecting the overall tag detection score. This 
a)
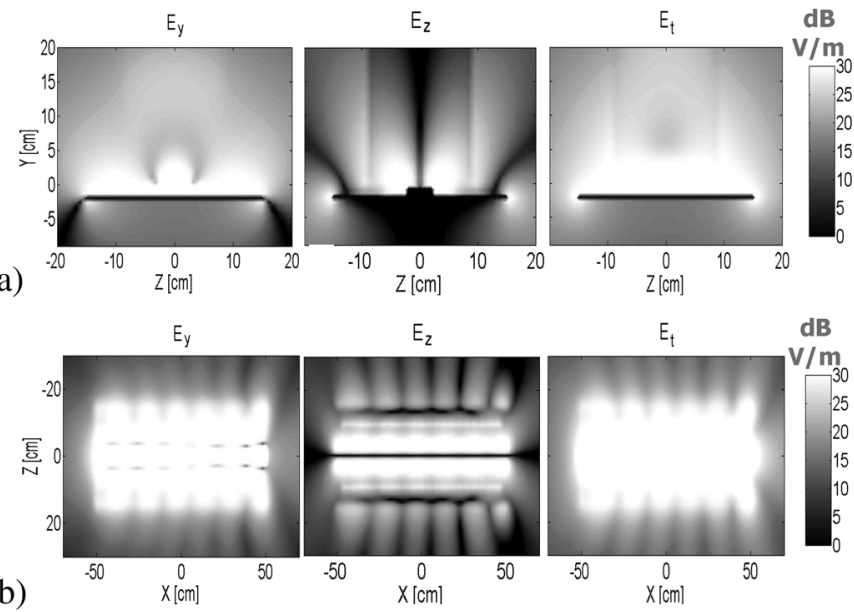

Fig. 5. Near-field components of the shelf with 21 books: (a) $X=0$ plane; (b) $Y=0$ plane (shelf surface)

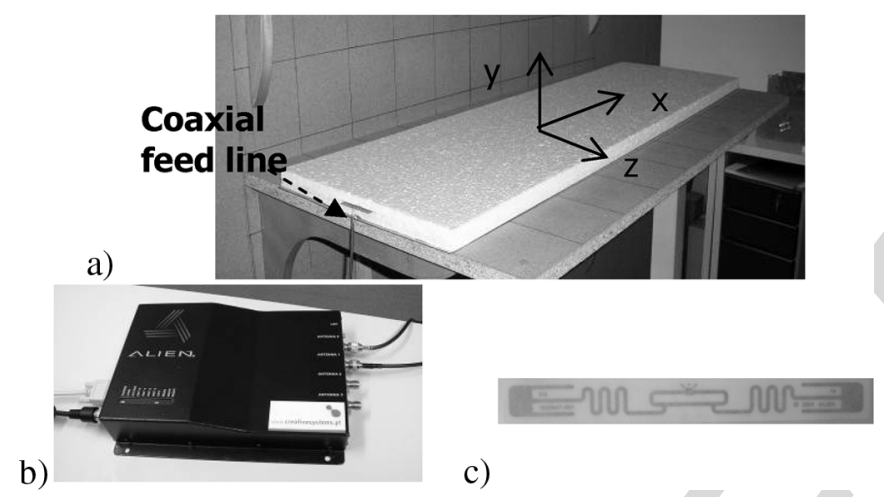

Fig. 6. Test environment: (a) fabricated shelf prototype; (b) ALIEN ALR-8800 reader; (c) ALIEN ALN-9540- "Squiggle" tag.

height is determined by the shape and lateral extent of the strip skewed path.

\section{EXPERIMENTAL RESULTS}

Fig. 6 shows the test environment used to evaluate the effectiveness of the proposed structure. The smart shelf is mounted on top of a preexisting wood structure [see Fig. 6(a)]. The ALIEN-ALR-8800 reader [7] in Fig. 6(b) is used to feed the proposed structure and its native software is used for score reading. The tags are ALIEN-ALN-9540_-"Squiggle" GEN 2 tag [7] [Fig. 6(c)]. The selected tag, with $9.7 \mathrm{~cm} \times 1.1 \mathrm{~cm}$ total size, is linear polarized and operates from 860 up to 920 MHz.

Measured and simulated input return loss of the embedded straight transmission line is presented in Fig. 7, both for the empty shelf and when it is fully loaded with 21 books. The transmission line is terminated with a matched load. The agreement between simulation and measurements is satisfactory. It is noted that when the 21 books are present, the line input return loss improves slightly, suggesting that more power is drained out from the line in this case.

Electric field components were measured near the empty shelf with the embedded microstrip line. The Agilent E8361A network analyzer was used in this test with a near-field probe a)

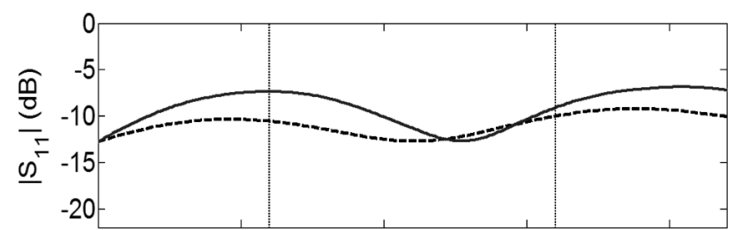

b)

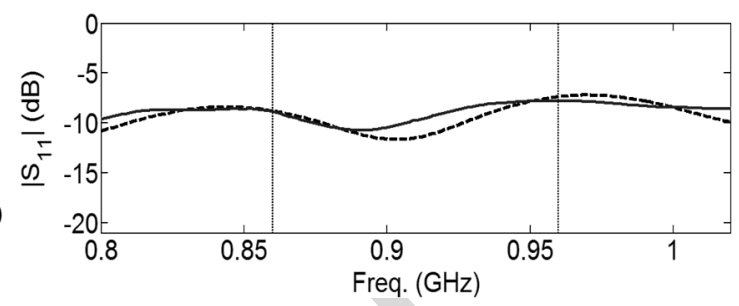

Fig. 7. Measured ( - ) and simulated (---) input impedance: (a) empty shelf; (b) shelf with 21 books.

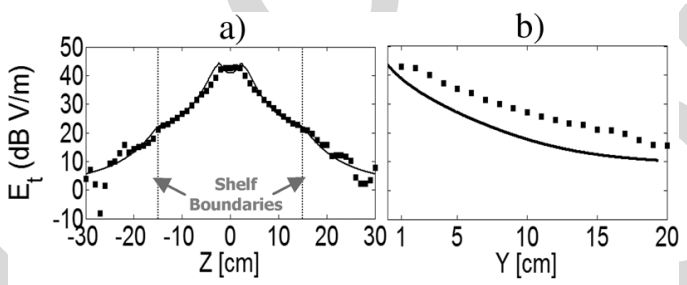

c)

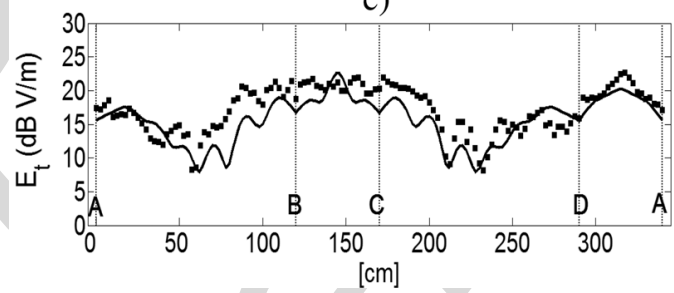

Fig. 8. Simulated $(-)$ and measured $(\cdots)$ total electrical near field in a shelf without books: (a) $x=0 \mathrm{~cm}, z=3 \mathrm{~cm}$ cut; (b) $y=0 \mathrm{~cm}, x=0 \mathrm{~cm}$ cut; (c) $y=0 \mathrm{~cm}, 10 \mathrm{~cm}$ away from the shelf over the ABCDA contour shown in Fig. 2(a).

scanning across two cut planes and along the shelf outside perimeter ABCDA (Fig. 2). Fig. 8 shows measured results superimposed on simulations: the agreement is quite good. Fig. 8(a) corresponds to $z$-axis scan $(x=0, y=0)$ and shows fast field decay outside the shelf, below the $20-\mathrm{dBV} / \mathrm{m}$ tag detection level. Fig. 8(b) corresponds to vertical coordinate scan at $(x=0, z=3)$ and anticipates tag detection up to 7 $\mathrm{cm}$. Fig. 8(c) corresponds to the closed contour of Fig. 2(a) for $x_{d}=10 \mathrm{~cm}$ : total electric field is maximum near the line feed and termination points, however field level is mainly below 20 $\mathrm{dBV} / \mathrm{m}$. In all cases, the experimental results follow quite well the simulation predictions.

For experimental confirmation of the RFID tag detection performance, the volume above the shelf was divided into a virtual lattice and an isolated tag was positioned at each node, according to prescribed orientations; the reading score was registered at each tag position.

The reading score was $100 \%$ for $z$-axis tag orientation over the shelf surface (Fig. 9). Although the $E_{z}$ component intensity is very low at the center of the microstrip line, this effect is very localized and does not deter reliable tag detection. The height above the shelf surface where the tag can be detected is 


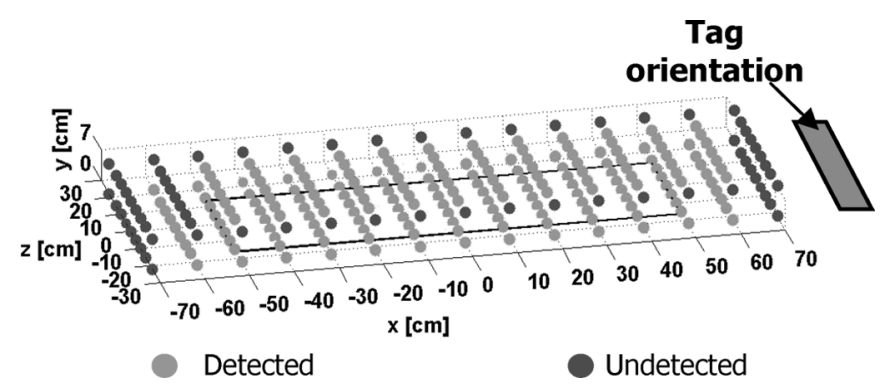

Fig. 9. Results of experimental detection of standalone tag positioned at each represented point, with $z$-axis orientation, without books.

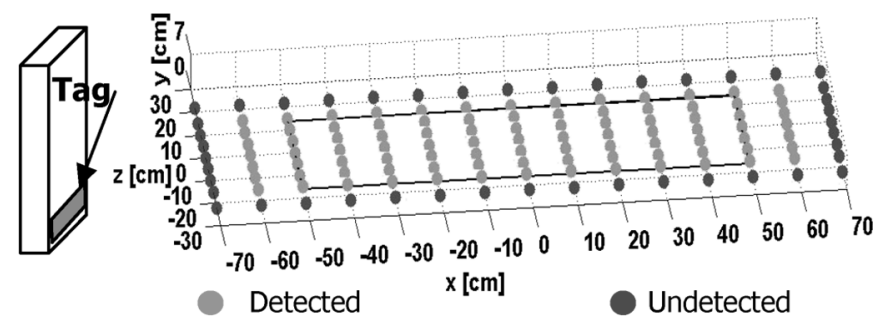

Fig. 10. Experimental detection of tags within books, oriented along $z$-axis.

controlled by adjusting the RF signal attenuation on the reader. For the straight microstrip line and no attenuation at the reader, $100 \%$ detection score is obtained up to $7 \mathrm{~cm}$ above the shelf surface, without spurious detection of tags placed on the shelf above or on the shelf below. The 7-cm maximum detection height is sufficient for the bookshelf application. Experimental results show that tag detection is obtained also for $x$-axis orientation in the regions above the microstrip line, where the other electric field components are also significant.

Similar tests were performed with the tags inserted into books, oriented along the $z$-axis. This is the preferred orientation to take advantage of the predominant and well confined $E_{z}$ field component. Simulations and experiment show that the presence of the book enhances the field intensity, favoring tag detection. Measurements were performed with a single book, with clusters of books, and with fully loaded shelf. Results for a single book are shown in Fig. 10. Each dot corresponds to each test position of the center of the book. It is seen that book detection is reasonably contained within the limits of the shelf. Experiments confirm that the lowest the tag placed inside the book is, the more reliable is the shelf reading capability. Similar results are obtained for fully loaded shelf.

Although the shelf design is intended for $z$-axis tag orientation, experiments showed that near $100 \%$ detection score is still possible when the tag inside the book is rotated in the $y-z$ plane. Even for the extreme $y$-axis tag orientation inside the book, detection is possible over most of the shelf surface, except for a region about $7 \mathrm{~cm}$ away from the center of the shelf [observable in Fig. 3(a)] where the intensity $E_{y}$ component is low and reading score may degrade.

So, books should be preferably placed vertically in the shelves with their tags at the bottom, oriented along the $z$-axis (transversal to the shelf). However, some flexibility is allowed in the tag orientation and height and detection is obtained as well for other book orientations on the shelf. These features favor practical use of the proposed solution.

\section{CONCLUSION}

This letter presents a new RFID low-cost smart shelf for book identification at UHF with proper confinement of the electromagnetic fields to avoid detection of books located outside the interrogated shelf, without the need of EM isolation barriers. The proposed approach uses the leaking fields of shaped microstrip transmission lines to enable proximity tags detection.

A prototype of the shelf was fabricated and tested using commercial reader and tags. Results demonstrate a very good match between experimental reading score and the simulated nearfield distribution; $100 \%$ reading score was achieved with the books and tags in the preferred orientations, but other orientations are also allowed which makes the proposed solution adequate for practical application on retail stores.

The presented smart shelf structure can be redesigned, without frequency restriction, to adjust for different shelf sizes and requirements. The transmission line circuit can be embedded on the shelf or mounted on top, for example, when metallic shelves are present, or above a preexisting structure.

Even though the presented work is focused on shelves and books, the concept has been successfully tested for other applications where tight detection confinement at UHF is required. For example, using meandered lines, it is possible to extend the detection height level and ensure detection for all tag orientations, which is attractive for shop cashier conveyors for automatic RFID reading of the product information as it slides over the device. It can be used also at isolated RFID reader points in the store. In summary, it can replace some functions that are presently performed with optical bar code manual readers, enabling a complete RFID-based solution in retail shop environment.

\section{ACKNOWLEDGMENT}

The authors would like to thank Creativesystems for discussions about the motivation and system specifications and for providing the RFID tags and reader equipment. They would also like to thank V. Fred and C. Brito for prototype fabrication and A. Almeida for prototype measurements.

\section{REFERENCES}

[1] X. Qing and Z. N. Chen, "Proximity effects of metallic environments on high frequency RFID reader antenna: Study and applications," IEEE Trans. Antennas Propag., vol. 55, no. 11, pp. 3105-3111, Nov. 2007.

[2] A. Cai, X. M. Qing, and Z. N. Chen, "High frequency RFID smart table antenna," Microw. Opt. Technol. Lett., vol. 49, no. 9, pp. 2074-2076, Sep. 2007.

[3] J. M. Lee, N. S. Kim, and C. S. Pyo, "A circular polarized metallic patch antenna for RFID reader," in Proc. Asia-Pacific Conf. Commun., Australia, Oct. 2005, pp. 116-118.

[4] Z.-M. Liu and R. R. Hillegass, "A 3 patch near field antenna for conveyor bottom read in RFID sortation application," in Proc. IEEE Antenna Propag. Soc. Int. Symp., Jul. 2006, pp. 1043-1046.

[5] D. M. Pozar, Microwave Engineering, 2nd ed. New York: Wiley, 1998.

[6] CST- Computer Simulation Tech. May 2008 [Online]. Available: http:// www.cst.com/

[7] ALIEN Technology May 2008 [Online]. Available: http://www.alientechnology.com 